\title{
A!
}

This is an electronic reprint of the original article.

This reprint may differ from the original in pagination and typographic detail.

Gladush, Yury; Mkrtchyan, Aram; Nyushkov, Boris; Ivanenko, Aleksey; Kokhanovskiy, Alexey; Kobtsev, Sergey; Nasibulin, Albert G.

Electro-optically gated in-line saturable absorbers for fibre lasers

Published in:

AOPC 2019

DOI:

$10.1117 / 12.2550646$

Published: 01/01/2019

Document Version

Publisher's PDF, also known as Version of record

Please cite the original version:

Gladush, Y., Mkrtchyan, A., Nyushkov, B., Ivanenko, A., Kokhanovskiy, A., Kobtsev, S., \& Nasibulin, A. G. (2019). Electro-optically gated in-line saturable absorbers for fibre lasers. In P. Zhou, J. Zhang, W. Li, S. Jiang, \& T. Taira (Eds.), AOPC 2019: Advanced Laser Materials and Laser Technology [113331M] (Proceedings of SPIE - The International Society for Optical Engineering; Vol. 11333). SPIE. https://doi.org/10.1117/12.2550646

This material is protected by copyright and other intellectual property rights, and duplication or sale of all or part of any of the repository collections is not permitted, except that material may be duplicated by you for your research use or educational purposes in electronic or print form. You must obtain permission for any other use. Electronic or print copies may not be offered, whether for sale or otherwise to anyone who is not an authorised user. 


\section{Electro-optically gated in-line saturable absorbers for fibre lasers}

Gladush, Yury, Mkrtchyan, Aram, Nyushkov, Boris, Ivanenko, Aleksey, Kokhanovskiy, Alexey, et al.

Yury Gladush, Aram Mkrtchyan, Boris Nyushkov, Aleksey Ivanenko, Alexey Kokhanovskiy, Sergey Kobtsev, Albert G. Nasibulin, "Electro-optically gated in-line saturable absorbers for fibre lasers," Proc. SPIE 11333, AOPC 2019: Advanced Laser Materials and Laser Technology, 113331M (18 December 2019); doi: 10.1117/12.2550646

SPE. Event: Applied Optics and Photonics China (AOPC2019), 2019, Beijing, China 


\title{
Electro-optically gated in-line saturable absorbers for fibre lasers
}

\author{
Yury Gladush ${ }^{1}$, Aram Mkrtchyan ${ }^{1}$, Boris Nyushkov, ${ }^{2,3}$, Aleksey Ivanenko ${ }^{2}$, Alexey Kokhanovskiy ${ }^{2}$, \\ Sergey Kobtsev ${ }^{2}$ and Albert G. Nasibulin ${ }^{1,4}$ \\ ${ }^{1}$ Skolkovo Institute of Science and Technology, 121205 Moscow, Russia \\ ${ }^{2}$ Novosibirsk State University, 630090 Novosibirsk, Russia \\ ${ }^{3}$ Novosibirsk State Technical University, 630073 Novosibirsk, Russia \\ ${ }^{4}$ Aalto University, 02150 Espoo, Finland
}

\begin{abstract}
This work presents for the first time a study of a fibre laser mode-locked due to a carbon-nanotube-based saturable absorber whose parameters could be controlled by a joined action of optical radiation and electric field. Combination of different types of control (optical and electrical) allowed variation of dynamics of the saturable absorber parameters and greater choice of pulsed generation regimes. We demonstrated the possibility of live switching of laser generation between various pulsed regimes through combined electro-optical action on the polymer-free carbon nanotube film.
\end{abstract}

Keywords: pulsed fibre laser; carbon-nanotube-based saturable absorber; electro-optical gating.

\section{INTRODUCTION}

Saturable absorbers are commonly used in lasers for mode locking and generation of ultra-short optical pulses [1-3]. Saturable absorbers based on carbon nanotubes are attractive thanks to their relative simplicity of fabrication and unique properties of nanotubes $[4,5]$. Application of carbon nano-tubes as saturable absorbers in fibre lasers [6-8] is also driven by their compatibility with all-fibre configurations of such lasers, in which the advantages of fibre-optical technologies are fully realised (no need for alignment or maintenance, etc).

Several recent publications [9-13] are dedicated to research into ways of dynamic modification by electric field or optical radiation of parameters of saturable absorbers made either with carbon nanotubes or noncarbon materials. Dynamic adjustment of saturable absorber parameters not only allows variation of the laser generation regime, but may also lead to a variety of generation regimes, thus providing a possibility of controllable switching between such different regimes. This expands substantially the functional capabilities of lasers, making them more flexible and universal sources of radiation.

Traditionally, carbon-nanotube saturable absorbers are composite materials containing a polymer matrix and carbon nano-tubes distributed in it [14]. Laser radiation may affect the properties of the polymer matrix; therefore it is desirable to use carbon nanotubers ether in a special matrix or without any matrix at all [15]. In the present work, we studied an erbium fibre laser with a saturable absorber made of polymer-free single-wall carbon nanotubes (SWCNTs), whose properties could be modified as a result of combined action of optical radiation and electric field. 


\section{EXPERIMENTAL SETUP}

The experimental installation used in this work is schematically presented in Fig. 1.

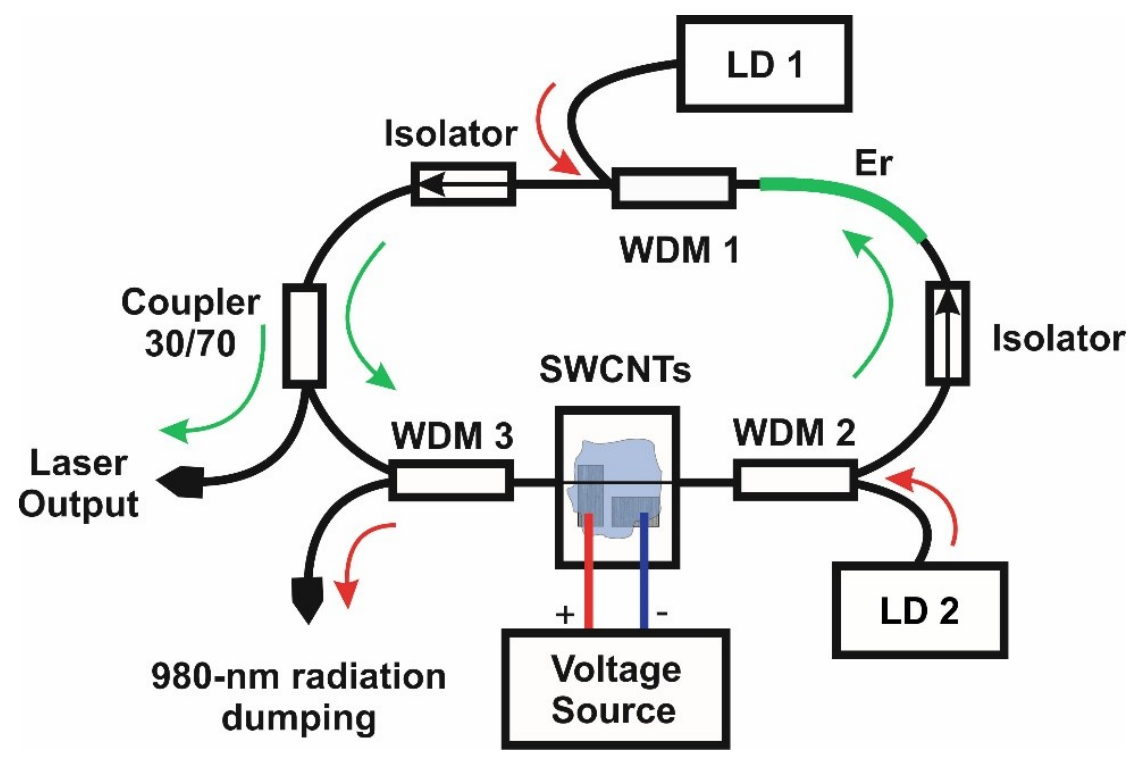

Fig. 1. Experimental setup: LD 1 - pump laser diode, LD 2 - laser diode for optical gating of SWCNTs; Er - Erdoped fibre; WDM 1, WDM 2, WDM 3 - wavelength division multiplexers.

The ring cavity of the Er-doped laser only used polarisation-maintaining (PM) elements. The role of the active medium was played by an $80-\mathrm{cm}$ long erbium-doped PM fibre pumped by a laser diode (LD 1) delivering up to $500 \mathrm{~mW}$ at $980 \mathrm{~nm}$. The resonator had two optical isolators, one of which ensures uni-directional laser operation and the other protects SWCNTs from the pump laser radiation. For optical modification of SWCNT parameters, an additional diode laser LD 2 was used, emitting up to $100 \mathrm{~mW}$ at $980 \mathrm{~nm}$. Radiation from this laser was guided into the cavity through multiplexer WDM 2 and left it through multiplexer WDM 3 after acting upon SWCNTs.

The erbium laser radiation interacted with SWCNTs via evanescent field accessible through a length of side-polished cavity fibre. On this polished side were deposited two 20-nm thick SWCNT stripes (Fig. 2) with a layer of ionic liquid between them. A voltage could be applied to these two stripes. The graph in Fig. 2 shows SWCNT transmission curves under optical (transmission curves \#1-3) and combined electro-optical (transmission curves \#4-6) action. It can be seen from this graph that combination of these two ways broadens the fibre transmittance range and therefore, also the range of saturable absorber parameters.

Shown in Fig. 3 are parameters of the generated pulses (duration, optical and radio-frequency spectra) in two regimes, which could be switched at will by adjustment of the electric potential applied to the SWCNT stripes (without any optical gating). The duration of pulses in mode-locked operation was equal to $800 \mathrm{fs}$, whereas in Q-switching mode, 1.1- $\mu$ s long pulses were generated. Mode-locked pulses were close to spectrally limited.

Fig. 4 presents parameters of the generated pulses at different radiation power levels applied to SWCNTs-SA (without any electric potential applied to SWCNT stripes). As the radiation power was raised to $60 \mathrm{~mW}$, the laser entered a regime of harmonic mode-locking. Raising the power of optical gating to $93 \mathrm{~mW}$ led to multi-pulse generation of the laser. Optical gating features faster regime switching as compared to electrical gating. Optical gating occurs due to the blooming response time of nano-tubes, which was equal to $200 \mathrm{fs}$ in this case. 

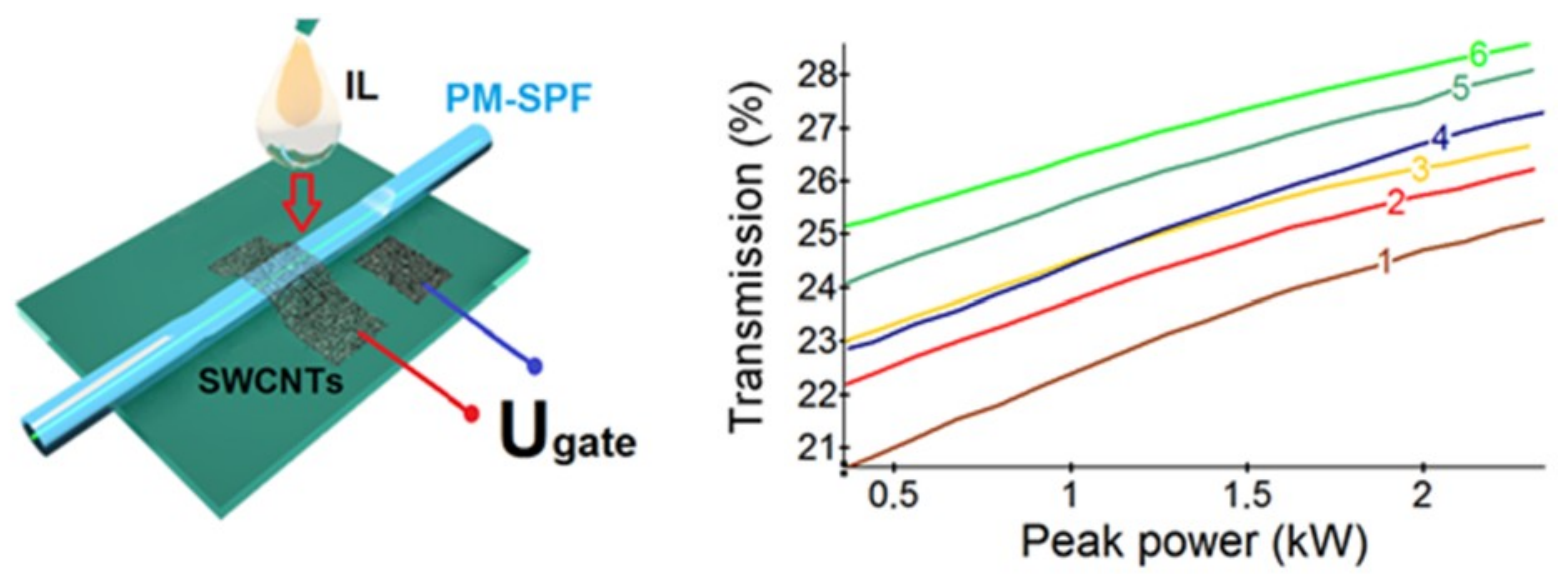

Fig. 2. Schematic diagram of the gated in-line SWCNTs-SA (left) and experimental curves of its nonlinear transmission at $1550 \mathrm{~nm}$ altered by the gate voltage and gating light at $980 \mathrm{~nm}$ (right). The transmission curves \#13 were obtained with zero gate voltage under the gating light power of $0 \mathrm{~mW}, 60 \mathrm{~mW}$, and $93 \mathrm{~mW}$, respectively. The transmission curves \#4-6 were obtained with the gate voltage of $1 \mathrm{~V}$ under the gating light power of $0 \mathrm{~mW}, 60$ $\mathrm{mW}$, and $93 \mathrm{~mW}$, respectively.

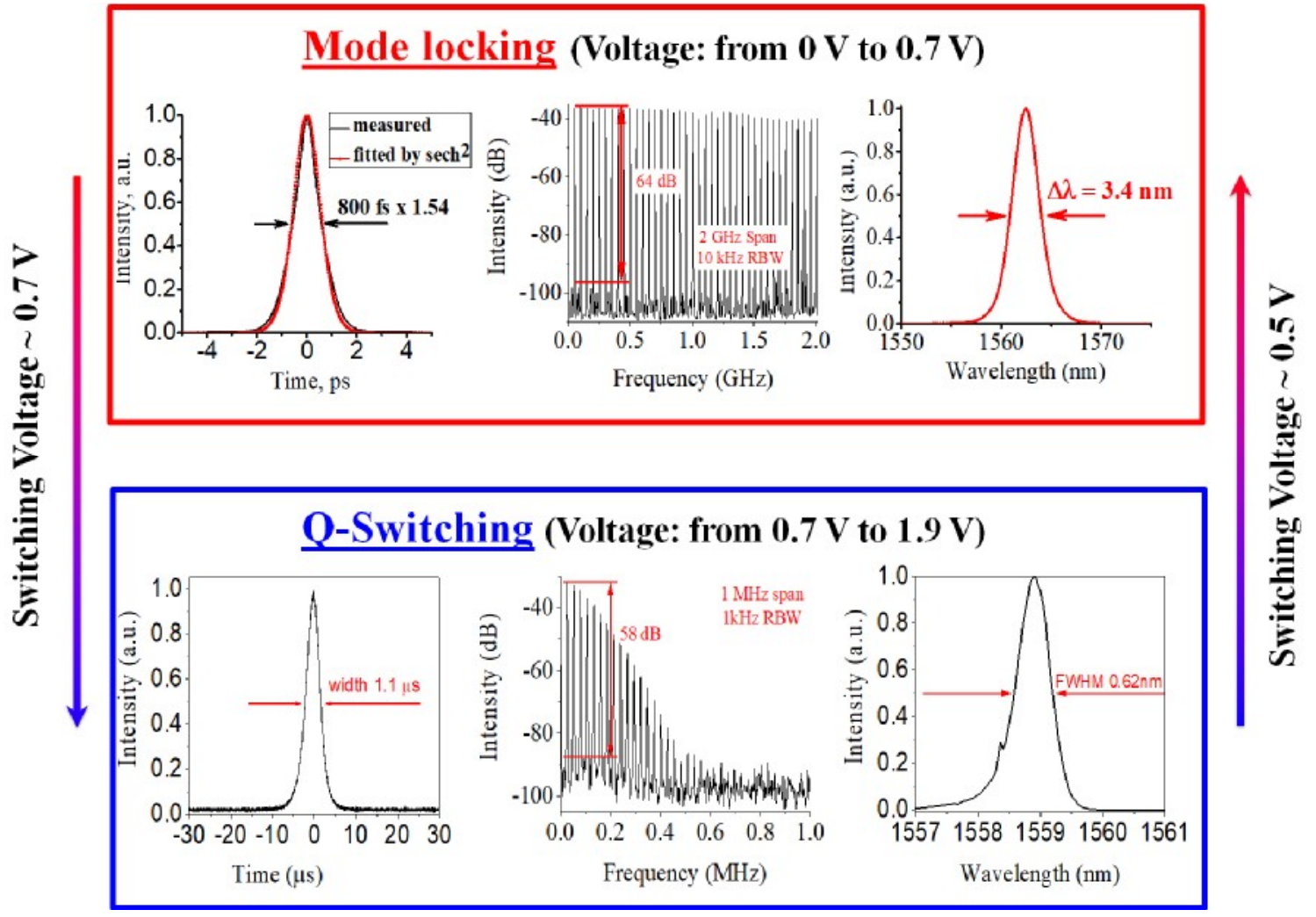

Fig. 3. Parameters of pulses generated in mode-locked (top) and Q-switched operation (bottom) in case of electrical gating applied to SWCNTs. 

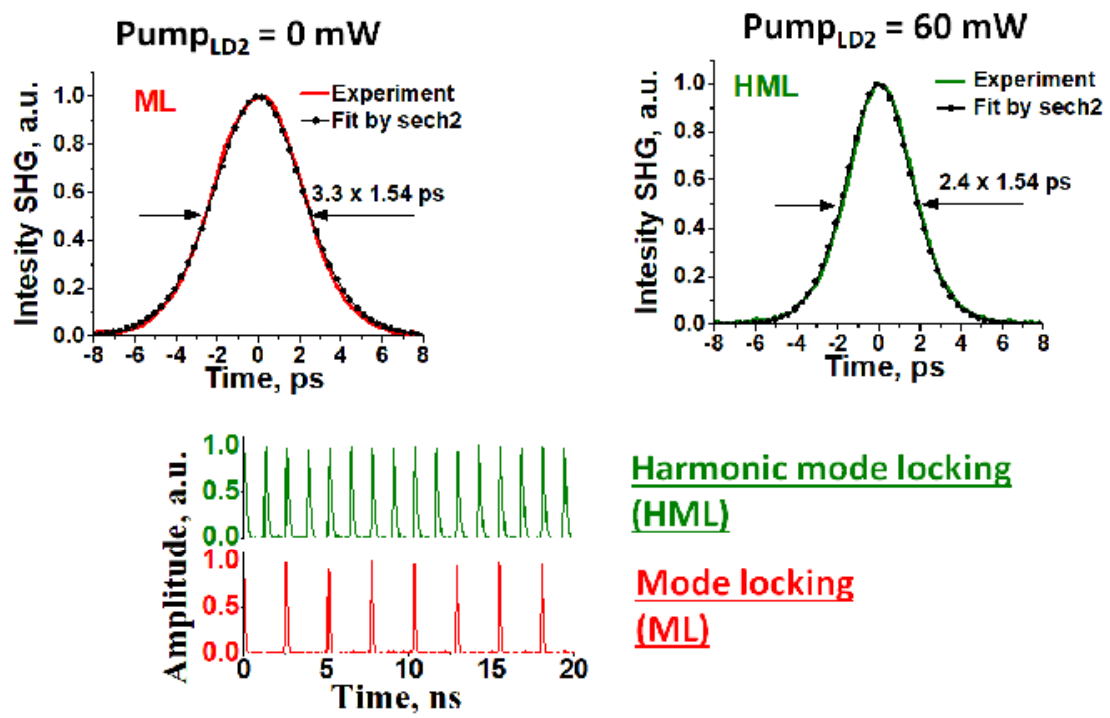

Harmonic mode locking

(HML)

Mode locking

$\underline{(M L)}$

Pump $_{\mathrm{LD} 2}=93 \mathrm{~mW}$
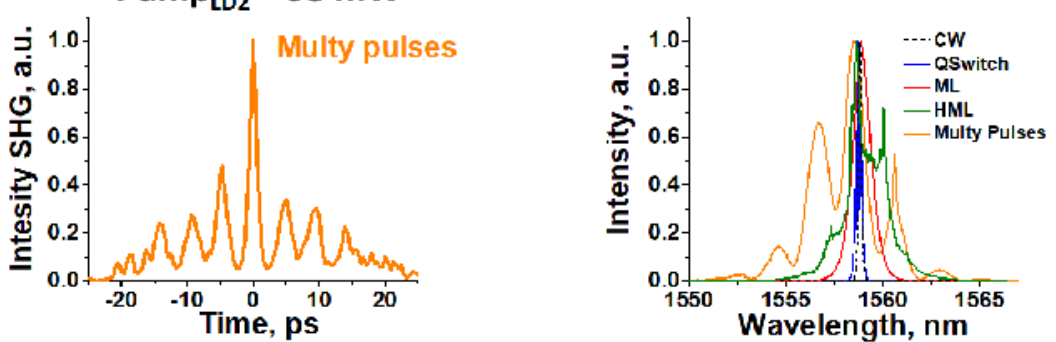

Fig. 4. Parameters of pulses generated at different power levels of radiation providing optical gating of SWCNTs-

SA.

\section{CONCLUSION}

Experimental study of an Er-doped fibre laser with properties of its carbon-nanotube saturable absorber modifiable due to combined action of optical radiation and electric field have shown that both these factors noticeably affect the parameters of laser generation and their combined action broadens the range of saturable absorber properties. The conducted research indicates a significant promise of combined control over the parameters of carbon-nanotube saturable absorbers, which extends noticeably the functional capabilities of fibre lasers. It is important to note that optical pumping may also be electronically controlled, therefore combined electro-optical action is fully electronically driven in this case. Such fully electronic control over generation parameters is optimal for fibre lasers designed according to the hands-off concept.

\section{ACKNOWLEDGMENTS}

This work was supported by Russian Science Foundation (RSF) (17-12-01281). 


\section{REFERENCES}

[1] Zambon, B., Tomasi, F., Hennequin, D., Arimondo, E., "Investigations of models and experimental studies of a stationary regime for a laser with a saturable absorber," Phys. Rev. A 40(7), 3782-3795 (1989).

[2] Reddy, K., "Applications of reverse saturable absorbers in laser science," Curr. Sci. 61(8), 520-526 (1991).

[3] Tutt, L., Boggess, T., "A review of optical limiting mechanisms and devices using organics, fullerenes, semiconductors and other materials," Prog. Quant. Electron. 17, 299-338 (1993).

[4] Avouris, P., Freitag, M., Perebeinos, V., "Carbon-nanotube photonics and optoelectronics," Nature Photonics 2, 341-350 (2008).

[5] Kivistö, S., Hakulinen,T., Kaskela, A., Aitchison, B., Brown, D., Nasibulin, A., Kauppinen, E., Härkönen, A., Okhotnikov, O., "Carbon nanotube films for ultrafast broadband technology," Opt. Express 17(4), 2358-2363 (2009).

[6] Kobtsev, S., Kukarin, S., Fedotov, Y., "Mode-locked Yb fiber laser with saturable absorber based on carbon nanotubes," Las. Phys. 21(2), 283-286 (2011).

[7] Martinez, A., Sun, Z., "Nanotube and graphene saturable absorbers for fibre lasers," Nature Photonics 7, 842845 (2013).

[8] Chernysheva, M., Rozhin, A., Fedotov, Y., Mou, C., Arif, R., Kobtsev, S., Dianov, E., Turitsyn, S., "Carbon nanotubes for ultrafast fibre lasers," Nanophotonics 6(1), 1-30 (2017).

[9] Gladush, Y., Mkrtchyan, A., Kopylova, D., Ivanenko, A., Nyushkov, B., Kobtsev, S., Kokhanovskiy, A., Khegai, A., Melkumov, M., Burdanova, M., Staniforth, M., Lloyd-Hughes, J., Nasibulin, A., "Ionic liquid gated carbon nanotube saturable absorber for switchable pulse generation," Nanoletters 19 (2019), in press. DOI: 10.1021/acs.nanolett.9b01012.

[10] Lee, E., Choi, S., Jeong, H., Park, ., Yim, W., Kim, M., Park, J., Son, S., Bae, S., Kim, S., Lee, K., Ahn, Y., Ahn, K., Hong, B., Park, J., Rotermund, F., Yeom, D., "Active control of all-fibre graphene devices with electrical gating," Nature Communications 6, 6851 (2015)

[11] Gene, J., Park, N., Jeong, H., Choi, S., Rotermund, F., Yeom, D., Kim, B., "Optically controlled in-line graphene saturable absorber for the manipulation of pulsed fiber laser operation," Opt. Express 24(19), 2130121307 (2016)

[12] Baylam, I., Özharar, S., Kakenov, N., Kocabaş, C., Sennaroğlu, A., "Femtosecond pulse generation with voltage-controlled graphene saturable absorbers," Chapter 12 (pp. 389-433) in book "Optical Properties of Graphene" (ed. R. Binder), World Scientific, 2017, 516p.

[13] Yao, B., Soavi, G., Ma, T., Zhang, X., Fu, B., Yoon, D., Hussain, S., Lombardo, A., Popa, D., Ferrari, A., "Gate controllable ultrafast fiber lasers based on graphene," CLEO-2018, San Jose, CA, OSA Technical Digest (online), paper SF3K.6.

[14] Khan, W., Sharma, R., Saini, R., "Carbon nanotube-based polymer composites: synthesis, properties and application," Chapter 1 (pp. 1-45) in book "Carbon Nanotubes. Current Progress of their Polymer Composites" (ed. M. Berber, I. Hafez), Intech, 2016, 504p.

[15] Kobtsev, S., Ivanenko, A., Gladush, Y., Nyushkov, B., Kokhanovskiy, A., Anisimov, A., Nasibulin, A., "Ultrafast all-fibre laser mode-locked by polymer-free carbon nanotube film," Opt. Express 24(25), 2876828773 (2016). 